\title{
Number of Points for Lambda z TAU
}

National Cancer Institute

\section{Source}

National Cancer Institute. Number of Points for Lambda z TAU. NCI Thesaurus. Code C147480.

The number of time points used in computing Lambda $z$ determined in a dosing interval. 